\title{
Quasi-states and symplectic intersections
}

\author{
Michael Entov* and Leonid Polterovich ${ }^{\dagger}$
}

\begin{abstract}
We establish a link between symplectic topology and a recently emerged branch of functional analysis called the theory of quasi-states and quasi-measures (also known as topological measures). In the symplectic context quasi-states can be viewed as an algebraic way of packaging certain information contained in Floer theory, and in particular in spectral invariants of Hamiltonian diffeomorphisms introduced recently by Yong-Geun Oh. As a consequence we prove a number of new results on rigidity of intersections in symplectic manifolds. This work is a part of a joint project with Paul Biran.
\end{abstract}

\section{Contents}

1 Introduction . . . . . . . . . . . . . . . . . 75

2 Results on symplectic intersections . . . . . . . . . . . . . . . 77

3 Quasi-states and quasi-measures . . . . . . . . . . . . . . . . 79

4 What happens on more general symplectic manifolds? . . . . . . . . . 81

5 Spectral numbers - review .................... 82

6 From a Calabi quasi-morphism to a symplectic quasi-state . . . . . . . . . 83

7 A partial symplectic quasi-state . . . . . . . . . . . . . . . . . . 84

8 Symplectic quasi-states on surfaces . . . . . . . . . . . . . . 88

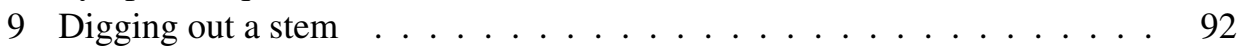

10 On the history and the physical meaning of quasi-states . . . . . . . . 94

\section{Introduction}

Rigidity of intersections is a class of phenomena in symplectic topology meaning that certain subsets of a symplectic manifold intersect each other in more points than dictated by algebraic and differential topology (see [11] for an excellent survey). In this paper we show that such rigidity phenomena in a closed symplectic manifold $M$

*Partially supported by the Technion President Fund, by the Israel Science Foundation grant \# 68/02 and by M. and C. Papo Research Fund.

${ }^{\dagger}$ Partially supported by the Israel Science Foundation grant \# 11/03. 
sometimes formally follow from the existence of real-valued functionals with some interesting algebraic properties on the Poisson algebra $C^{\infty}(M)$.

On the one hand, these functionals are related to the notions of quasi-state and quasi-measure (which have been recently called topological measures) on $M$ (see Section 3) which originate in quantum mechanics [1], [2] and have been a subject of intensive study in recent years following the paper [3] by J. F. Aarnes.

On the other hand, they are linked to a group-theoretic notion of quasi-morphism (see e.g. [29]) which already appeared in the context of symplectic topology in [20], [13]. The symplectic quasi-states on the Poisson-Lie algebra of functions on certain symplectic manifolds $M$ considered below arise as an infinitesimal version of the Calabi quasi-morphism introduced in [20]. This quasi-morphism is defined on the universal cover $\widetilde{\operatorname{Ham}}(M)$ of the group $\operatorname{Ham}(M)$ of Hamiltonian diffeomorphisms of $M$.

All the above-mentioned functionals are constructed by means of Floer theory for Hamiltonian flows on $M$ and can be viewed as an algebraic way of packaging certain information contained in that theory.

Throughout the paper $M$ always stands for a closed connected symplectic manifold with a symplectic form $\omega$. For technical reasons we assume that $M$ is rational, i.e. the image of $\pi_{2}(M)$ under the cohomology class of $\omega$ is a discrete subgroup of $\mathbb{R}$. Furthermore, we assume that $M$ is strongly semi-positive, that is

$$
2-n \leq c_{1}(A)<0 \Longrightarrow \omega(A) \leq 0, \quad \text { for any } A \in \pi_{2}(M),
$$

where $c_{1}$ stands for the 1 st Chern class of $(M, \omega)$. For instance, every symplectic 4-manifold is strongly semi-positive. Another interesting class of examples is given by spherically monotone symplectic manifolds, which means that $\left.[\omega]\right|_{\pi_{2}(M)}$ is a positive multiple of $\left.c_{1}\right|_{\pi_{2}(M)}$. Note that this condition automatically implies strong semi-positivity and rationality of $M$.

Organization of the paper. The next section contains our main results on symplectic intersections. In Section 3 we focus on a special class of symplectic manifolds $M$ which, for instance, includes monotone products of complex projective spaces. After a brief review of quasi-states and quasi-measures, we introduce symplectic quasistates on the algebra $C(M)$ which turn out to be useful for symplectic intersections in $M$. In Section 4 we present a weaker notion of a partial symplectic quasi-state and its applications to non-displaceability phenomenon on more general symplectic manifolds. In Section 5 we review spectral invariants of Hamiltonian diffeomorphisms introduced recently by Y.-G. Oh. In Sections 6 and 7 these invariants are used in order to construct the above-mentioned (partial) symplectic quasi-states. In Section 8 we discuss symplectic quasi-states on surfaces. The reader will see that some innocently looking basic questions in this direction require more advanced tools of the theory of quasi-states and quasi-measures. Section 9 contains some applications (in the spirit 
of our paper [13] with P. Biran) of our results to the Lagrangian intersection problem. In Section 10 we discuss the history and the physical meaning of quasi-states. In addition, in Sections 8-10 we present a number of open problems.

\section{Results on symplectic intersections}

We say that a subset $X$ of $M$ is displaceable if there exists a Hamiltonian diffeomorphism $\phi \in \operatorname{Ham}(M)$ so that

$$
\phi(X) \cap \operatorname{Closure}(X)=\emptyset .
$$

Otherwise, we call $X$ non-displaceable. For instance, an open hemisphere in $\mathbb{S}^{2}$ with the Euclidean area form is displaceable while the closed hemisphere is not.

A linear subspace $\mathcal{A} \subset C^{\infty}(M)$ is called Poisson-commutative, if $\{F, G\}=0$ for all $F, G \in \mathcal{A}$, where $\{\cdot, \cdot\}$ stand for the Poisson brackets. Given a finite-dimensional Poisson-commutative subspace $\mathcal{A} \subset C^{\infty}(M)$, its moment map $\Phi_{\mathcal{A}}: M \rightarrow \mathcal{A}^{*}$ is defined as

$$
\left\langle\Phi_{\mathcal{A}}(x), F\right\rangle=F(x) .
$$

Non-empty subsets of the form $\Phi_{\mathcal{A}}^{-1}(p), p \in \mathcal{A}^{*}$, are called fibers of $\mathcal{A}$.

Theorem 2.1. Any finite-dimensional Poisson-commutative subspace of $C^{\infty}(M)$ has at least one non-displaceable fiber. Moreover, if every fiber has a finite number of connected components, there exists a fiber with a non-displaceable connected component.

Poisson-commutative subspaces naturally appear when $M$ is equipped with the structure of a (singular) Lagrangian fibration. In this case Theorem 2.1 shows that the fibration has at least one non-displaceable fiber. For instance, we have the following corollary, where the fibration is given by the moment map of a Hamiltonian torus action.

Corollary 2.2. Assume that $M^{2 n}$ is equipped with a Hamiltonian action of $\mathbb{T}^{n}$. Then at least one Lagrangian orbit of this action is non-displaceable.

Proof. Let $\mathcal{A}$ be the span of the coordinate functions associated to the moment map of the action. Every fiber of $\mathcal{A}$ is a fiber of the moment map: it is either a Lagrangian torus, or an isotropic torus of dimension less than $n$. The latter are displaceable (see e.g. [12]). Hence the result follows immediately from Theorem 2.1.

Definition 2.3. A closed subset $X \subset M$ is called a stem, if there exists a finitedimensional Poisson-commutative subspace $\mathcal{A} \subset C^{\infty}(M)$ so that $X$ is a fiber of $\mathcal{A}$ and each fiber of $\mathcal{A}$, other than $X$, is displaceable. 
Note that the image of a stem under any symplectomorphism of $M$ is again a stem.

Theorem 2.1 guarantees that every stem is non-displaceable. This result can be strengthened for a special class of symplectic manifolds as follows.

Theorem 2.4. Suppose $M$ is one of the following symplectic manifolds: $\mathbb{C P}^{n}, a$ complex Grassmannian, $\mathbb{C P}^{n_{1}} \times \cdots \times \mathbb{C P}^{n_{k}}$ with a monotone product symplectic structure, the monotone symplectic blow-up of $\mathbb{C P}^{2}$ at one point. Then any two stems in $M$ have an non-empty intersection.

In particular, a stem in such an $M$ cannot be displaced from itself by any (not necessarily Hamiltonian) symplectomorphism.

Here is a sample corollary of this theorem. Consider the 2-sphere $\mathbb{S}^{2}$ with a symplectic form $\omega$ of total area 1 . Define a class $g_{\mathbb{S}^{2}}$ of closed subsets $\Gamma \subset \mathbb{S}^{2}$ with the following property: The complement $\mathbb{S}^{2} \backslash \Gamma$ has a finite number of connected components, and each of them is homeomorphic to a disc and has area $\leq \frac{1}{2}$. For instance, one can take an equator, or the 1-skeleton of a piecewise smooth triangulation of $\mathbb{S}^{2}$ with small enough 2-dimensional faces.

Corollary 2.5. Let $M$ be the direct product of $m$ copies of $\left(\mathbb{S}^{2}, \omega\right)$ and let $\Gamma_{i}, \Gamma_{i}^{\prime} \in$ $\mathcal{Q}_{\mathbb{S}^{2}}, i=1, \ldots, m$. Then the subsets $\Gamma_{1} \times \cdots \times \Gamma_{m}$ and $\phi\left(\Gamma_{1}^{\prime} \times \cdots \times \Gamma_{m}^{\prime}\right)$ have a non-empty intersection for every symplectomorphism $\phi$ of $M$.

Proof. Note that a direct product of stems is a stem. Hence it suffices to verify that every $\Gamma \in g_{\mathbb{S}^{2}}$ is a stem. Let $U_{1}, \ldots, U_{d}$ be the connected components of $\mathbb{S}^{2} \backslash \Gamma$.

Take smooth functions $H_{1}, \ldots, H_{d}$ as follows: $H_{i}$ vanishes on $\mathbb{S}^{2} \backslash U_{i}$ and $H_{i}$ is strictly positive on $U_{i}$. The existence of such $H_{1}, \ldots, H_{d}$ follows easily from the fact that any closed subset of $\mathbb{R}^{2}$ is the zero-level set of some smooth real-valued function on $\mathbb{R}^{2}$ (see e.g. [35], Lemma 1.4.13).

Put $\mathcal{A}=\operatorname{Span}_{\mathbb{R}}\left(H_{1}, \ldots, H_{d}\right)$. Clearly $\mathcal{A}$ is Poisson-commutative and $\Gamma=$ $\Phi_{A}^{-1}(0)$ is its fiber. All other fibers are closed subsets of one of the $U_{i}$ 's, and hence are displaceable. Therefore $\Gamma$ is a stem and the result follows from Theorem 2.4.

Here is another corollary of Theorem 2.1. Let $\mathbb{T}^{2}$ be a torus with coordinates $p, q \in \mathbb{R} / \mathbb{Z}$ and the symplectic form $d p \wedge d q$. Equip $M \times \mathbb{T}^{2}$ with the product symplectic structure and assume that the resulting symplectic manifold is strongly semi-positive and rational. Denote by $S$ a meridian $p=$ const of $\mathbb{T}^{2}$.

Corollary 2.6. Assume $X \subset M$ is a stem. Then $X \times S \subset M \times \mathbb{T}^{2}$ is non-displaceable.

Proof. Let $\mathcal{A} \subset C^{\infty}(M)$ be a finite-dimensional Poisson-commutative subspace such that the stem $X$ is its only non-displaceable fiber. Lift to $M \times \mathbb{T}^{2}$ the functions on $M$ that belong to $\mathcal{A}$ as well as the functions $\sin 2 \pi p, \cos 2 \pi p$ on $\mathbb{T}^{2}$. All these lifts 
together span a Poisson-commutative subspace $\mathcal{A}^{\prime} \subset C^{\infty}\left(M \times \mathbb{T}^{2}\right)$ such that each of its fibers is a direct product of a fiber of $\mathcal{A}$ and a meridian of $\mathbb{T}^{2}$.

Theorem 2.1 says that $\mathcal{A}^{\prime}$ must have a non-displaceable fiber $Y$. Since $X$ is the only non-displaceable fiber of $\mathcal{A}$, the fiber $Y$ has to have the form $Y=X \times S^{\prime}$ for some meridian $S^{\prime}$ of $\mathbb{T}^{2}$. But any two meridians of $\mathbb{T}^{2}$ can be mapped into each other by a symplectomorphism of $\mathbb{T}^{2}$ - hence the products of these meridians with $X$ can be mapped into each other by a symplectomorphism of $M \times \mathbb{T}^{2}$. Thus if $X \times S^{\prime}$ is non-displaceable, then $X \times S$ has to be non-displaceable as well.

\section{Quasi-states and quasi-measures}

Write $C(M)$ for the commutative (with respect to multiplication) Banach algebra of all continuous functions on $M$ endowed with the uniform norm. For a function $F \in C(M)$ denote by $\mathcal{A}_{F}$ the uniform closure of the set of functions of the form $p \circ F$, where $p$ is a real polynomial. A (not necessarily linear) functional $\zeta: C(M) \rightarrow \mathbb{R}$ is called a quasi-state [3], if it satisfies the following axioms:

Quasi-linearity. $\zeta$ is linear on $\mathcal{A}_{F}$ for every $F \in C(M)$ (in particular $\zeta$ is homogeneous).

Monotonicity. $\zeta(F) \leq \zeta(G)$ for $F \leq G$.

Normalization. $\zeta(1)=1$.

A quasi-state is called symplectic, if it has the following additional properties:

Strong quasi-additivity. $\zeta(F+G)=\zeta(F)+\zeta(G)$ for all smooth functions $F, G$ which commute with respect to the Poisson bracket: $\{F, G\}=0$.

Vanishing. $\zeta(F)=0$, provided supp $F$ is displaceable.

Symplectic invariance. $\zeta(F)=\zeta(F \circ f)$ for every symplectic diffeomorphism $f \in \operatorname{Symp}_{0}(M)$ (here $\operatorname{Symp}_{0}(M)$ stands for the identity component of the group $\operatorname{Symp}(M)$ of symplectomorphisms).

Note that strong quasi-additivity together with homogeneity yields quasi-linearity. Indeed, if $F$ is smooth, $\left\{p_{1} \circ F, p_{2} \circ F\right\}=0$ for every pair of polynomials $p_{1}$ and $p_{2}$. Observing that $\zeta$ is continuous in the uniform topology because of the monotonicity and normalization axioms, one can easily extend the result for a general continuous $F$.

Theorem 3.1. Suppose $M$ is one of the following symplectic manifolds: $\mathbb{C P}^{n}, a$ complex Grassmannian, $\mathbb{C P}^{n_{1}} \times \cdots \times \mathbb{C P}^{n_{k}}$ with a monotone product symplectic structure, the monotone symplectic blow-up of $\mathbb{C P}^{2}$ at one point. Then $C(M)$ admits a symplectic quasi-state. 
In [3] Aarnes proved a generalized Riesz representation theorem which associates to each quasi-state $\zeta$ a quasi-measure $\tau_{\zeta}$, that is a "measure" which is finitely additive but not necessarily sub-additive. More precisely, denote by $\&$ the collection of all subsets of $M$ which are either open or closed. A quasi-measure (recently called a topological measure in the literature) on $M$ is a $[0,1]$-valued set-function $\tau$ on $\&$ such that

1) $\tau(M)=1$

2) $X_{1} \subset X_{2} \Rightarrow \tau\left(X_{1}\right) \leq \tau\left(X_{2}\right)$ for all $X_{1}, X_{2} \in$ s;

3) $\tau\left(X_{1} \sqcup \cdots \sqcup X_{k}\right)=\tau\left(X_{1}\right)+\cdots+\tau\left(X_{k}\right)$ for all $X_{1}, \ldots, X_{k} \in \&$ with $X_{1} \sqcup \cdots \sqcup X_{k} \in \&$;

4) for every open subset $X$ one has $\tau(X)=\sup \tau(A)$, where the supremum is taken over all closed subsets $A \subset X$.

The relation between a quasi-state $\zeta$ and the corresponding quasi-measure $\tau_{\zeta}$ is the following ([3]). Given a closed $X \subset M$, consider the set $\mathcal{F}_{X}$ of smooth functions $M \rightarrow[0,1]$ which are identically equal to 1 on $X$. A quasi-state $\zeta$ is bounded on $\mathcal{F}_{X}$ by 0 and 1 and therefore one can define

$$
\tau_{\zeta}(X):=\inf _{F \in \mathcal{F}_{X}} \zeta(F)
$$

Intuitively, $\tau_{\zeta}(X)$ is the "value" of the functional $\zeta$ on the (discontinuous) characteristic function of $X$. For an open subset $Y$ put $\tau_{\zeta}(Y)=1-\tau_{\zeta}(M \backslash Y)$.

Lemma 3.2. Assume a closed connected symplectic manifold $M$ admits a symplectic quasi-state $\zeta$. Denote by $\tau$ the corresponding quasi-measure. Then $\tau(X)=1$ for every stem $X \subset M$.

Proof. Let $\mathcal{A} \subset C^{\infty}(M)$ be a finitely generated Poisson-commutative subspace. Denote by $\Delta \subset \mathcal{A}^{*}$ the image of the moment map $\Phi_{\mathcal{A}}$. Write $C_{0}^{\infty}\left(\mathcal{A}^{*}\right)$ for the space of all smooth compactly supported functions on $\mathcal{A}^{*}$. Note that the functional

$$
I: C_{0}^{\infty}\left(\mathcal{A}^{*}\right) \rightarrow \mathbb{R}, \quad G \mapsto \zeta\left(\Phi_{\mathcal{A}}^{*} G\right),
$$

is a positive distribution ${ }^{1}$ (use the strong quasi-additivity and the monotonicity axioms of $\zeta$ ). Hence it defines a measure $\sigma$ on $\mathcal{A}^{*}$ so that $I(G)=\int_{\mathcal{A}^{*}} G d \sigma$ (see e.g. [22], Ch. 2, Sec. 2). By the normalization axiom, $\sigma$ is a probability measure. Obviously, supp $\sigma \subset \Delta$. The vanishing axiom yields that if $\Phi_{\mathcal{A}}^{-1}(p)$ is displaceable for some $p \in \Delta$, then $p \notin \operatorname{supp} \sigma$. Thus, if $X=\Phi_{\mathcal{A}}^{-1}\left(p_{0}\right)$ is a stem associated to $\mathcal{A}$, the measure $\sigma$ must be the Dirac measure at $p_{0}$. Using this and considering in the definition of $\tau(X)$ the functions $F \in \mathcal{F}_{X}$ of the form $F=\Phi_{\mathcal{A}}^{*} G, G \in C_{0}^{\infty}\left(\mathcal{A}^{*}\right)$, one readily gets $\tau(X)=1$.

\footnotetext{
${ }^{1}$ Recall that a distribution (that is a continuous linear functional) on $C_{0}^{\infty}\left(\mathbb{R}^{N}\right)$ is called positive if it takes non-negative values on non-negative functions.
} 
The proof of the lemma shows that if $\tau$ is a quasi-measure defined by a symplectic quasi-state, and $\mathcal{A} \subset C^{\infty}(M)$ is a finitely generated Poisson-commutative subspace, the push-forward of $\tau$ by the moment map $\Phi_{\mathcal{A}}$ is a genuine measure on the image of $\Phi_{\mathcal{A}}$. In case when $\tau$ comes from a quasi-state which is not strongly quasi-additive (and thus not symplectic), this may no longer be true and moreover such a quasi-measure may vanish on a stem - see Remark 8.4.

Proof of Theorem 2.4 (assuming Theorem 3.1). According to Theorem 3.1, any $M$ mentioned in the hypothesis of Theorem 2.4 admits a symplectic quasi-state. Let $\tau$ be the corresponding quasi-measure and let $X, Y \subset M$ be stems. Lemma 3.2 implies that $\tau(X)=\tau(Y)=1$. If $X$ and $Y$ do not intersect, we have $\tau(X \cup Y)=$ $\tau(X)+\tau(Y)=1+1=2$, and we get a contradiction with $\tau(X \cup Y) \leq \tau(M)=1$.

\section{What happens on more general symplectic manifolds?}

Let $\zeta: C(M) \rightarrow \mathbb{R}$ be a (not necessarily quasi-linear) functional which satisfies monotonicity, normalization, vanishing and invariance axioms from the previous section. Assume that it has two additional properties:

Partial additivity. If $F_{1}, F_{2} \in C^{\infty}(M),\left\{F_{1}, F_{2}\right\}=0$ and the support of $F_{2}$ is displaceable, then $\zeta\left(F_{1}+F_{2}\right)=\zeta\left(F_{1}\right)$.

Semi-homogeneity. $\zeta(\lambda F)=\lambda \zeta(F)$ for any $F$ and any $\lambda \in \mathbb{R}_{\geq 0}$.

We call $\zeta$ a partial symplectic quasi-state.

Theorem 4.1. Let $M$ be a strongly semi-positive and rational closed connected symplectic manifold. Then $C(M)$ admits a partial symplectic quasi-state.

Theorem 4.1 will be proved in Section 7.

Proof of Theorem 2.1 (assuming Theorem 4.1). Let $\zeta$ be a partial symplectic quasistate. Assume on the contrary that all fibers of $\mathcal{A}$ are displaceable. Choose an open covering $U:=\left\{U_{1}, \ldots, U_{d}\right\}$ of the image $\Delta$ of the moment map $\Phi_{\mathcal{A}}$ so that the preimages $\Phi^{-1}\left(U_{i}\right)$ are displaceable. Let $\rho_{1}, \ldots, \rho_{d}$ be a partition of unity associated to $U$, that is supp $\rho_{i} \subset U_{i}$ and $\left.\sum_{i=1}^{d} \rho_{i}\right|_{\Delta}=1$. Note that $\zeta\left(\Phi^{*} \rho_{i}\right)=0$ by vanishing property. Using the normalization and the partial additivity, we get

$$
1=\zeta(1)=\zeta\left(\sum_{i=1}^{d} \Phi^{*} \rho_{i}\right)=\sum_{i=1}^{d} \zeta\left(\Phi^{*} \rho_{i}\right)=0,
$$

and we get a contradiction. 
A similar argument shows that, if any fiber of $\mathcal{A}$ has a finite number of connected components, then at least one connected component of some fiber of $\mathcal{A}$ has to be non-displaceable.

\section{Spectral numbers - review}

We review a few basic facts about the spectral numbers of Hamiltonian diffeomorphisms introduced by Yong-Geun Oh [36] (see also [42], [41] for earlier versions of this theory). For the precise definitions and further details see [36], [20] and [34]. We assume here that $M$ is strongly semi-positive and rational. The strong semipositivity of $M$ is needed to guarantee that the moduli spaces of pseudo-holomorphic curves involved in the definitions of Floer and quantum homology and the isomorphism between them are well-behaved. In view of the developments [21], [30], [31], [32], concerning Floer theory for general symplectic manifolds, it is likely that the strong semi-positivity of $M$ is not essential for the existence of spectral numbers. The assumption that $M$ is rational is needed to guarantee the spectrality property below ${ }^{2}$, though it is likely that eventually this assumption will also be removed, see [37].

By $\overline{\operatorname{spec}}(H)$ we denote the action spectrum of a Hamiltonian $H$. Recall that it is the set of critical values of the action functional defined by $H$ on the universal cover of the space of free contractible loops in $M$.

A time-dependent Hamiltonian $H: M \times \mathbb{S}^{1} \rightarrow \mathbb{R}$ is called normalized if

$$
\int_{M} H(\cdot, t) \omega^{n}=0 \quad \text { for all } t \in \mathbb{S}^{1} .
$$

It turns out that $\overline{\operatorname{spec}}\left(H_{1}\right)=\overline{\operatorname{spec}}\left(H_{2}\right)$ for any normalized $H_{1}, H_{2}$ generating the same element $\phi \in \widetilde{\operatorname{Ham}}(M)$. Thus one can define $\operatorname{spec}(\phi)$ for any $\phi \in \widetilde{\operatorname{Ham}}(M)$ as $\overline{\operatorname{spec}}(H)$ for any normalized $H$ generating $\phi$.

Denote by $Q H_{*}(M)$ the quantum homology ring of $M$ (with coefficients in $\mathbb{C}$ ) and by $*$ the product in that ring. The fundamental class $[M]$ is the unit in the ring. To each non-zero quantum homology class $a \in Q H_{*}(M)$ and each time-dependent Hamiltonian $H: M \times \mathbb{S}^{1} \rightarrow \mathbb{R}$ one can associate a spectral number $\bar{c}(a, H)$. Spectral numbers have the following properties which are relevant for us:

Spectrality. $\bar{c}(a, H) \in \overline{\operatorname{spec}}(H)$.

Shift property. $\bar{c}(a, H+\lambda(t))=\bar{c}(a, H)+\int_{0}^{1} \lambda(t) d t$ for any Hamiltonian $H$ and function $\lambda: \mathbb{S}^{1} \rightarrow \mathbb{R}$.

Monotonicity. If $H_{1} \leq H_{2}$, then $\bar{c}\left(a, H_{1}\right) \leq \bar{c}\left(a, H_{2}\right)$.

\footnotetext{
${ }^{2}$ For the same reason the rationality assumption should be added to the results $2.5 .3,2.5 .4,2.6 .1$ and to part 4 of 2.4.2 in [19] which involve the spectral numbers.
} 
Lipschitz property. The map $H \mapsto \bar{c}(a, H)$ is Lipschitz on the space of (timedependent) Hamiltonians $H: M \times \mathbb{S}^{1} \rightarrow \mathbb{R}$ with respect to the $C^{0}$-norm.

Symplectic invariance. $\bar{c}\left(a, \phi^{*} H\right)=\bar{c}(a, H)$ for every $\phi \in \operatorname{Symp}_{0}(M), H \in$ $C^{\infty}(M)$.

Normalization. $\bar{c}(a, 0)=0$ for every even-dimensional singular homology class $a \in H_{*}(M, \mathbb{C})$.

Homotopy invariance. $\bar{c}\left(a, H_{1}\right)=\bar{c}\left(a, H_{2}\right)$ for any normalized $H_{1}, H_{2}$ generating the same $\phi \in \widetilde{\operatorname{Ham}}(M)$. Thus one can define $c(a, \phi)$ for any $\phi \in \widetilde{\operatorname{Ham}}(M)$ as $\bar{c}(a, H)$ for any normalized $H$ generating $\phi$. Note that $c(a, \phi) \in \operatorname{spec}(\phi)$.

Triangle inequality. $c(a * b, \phi \psi) \leq c(a, \phi)+c(b, \psi)$.

\section{From a Calabi quasi-morphism to a symplectic quasi-state}

In this section we prove Theorem 3.1. Assume that $M$ is spherically monotone. In this case the Novikov ring of $M$ is a field of complex Laurent series in one variable. The even-degree part $Q H_{\mathrm{ev}}(M)$ of $Q H_{*}(M)$ is a commutative algebra over this field. Assume that the algebra $Q H_{\mathrm{ev}}(M)$ is semi-simple in the sense of [20] - this holds, for instance, if $M$ is one of the symplectic manifolds listed in the statement of Theorem 3.1: the standard $\mathbb{C P}^{n}$, a complex Grassmannian, $\mathbb{C P}^{n_{1}} \times \cdots \times \mathbb{C P}^{n_{k}}$ with a monotone product symplectic structure, the monotone symplectic blow-up of $\mathbb{C P}^{2}$ at one point. Denote by $\operatorname{vol}\left(M^{2 n}\right):=\int_{M} \omega^{n}$ the total symplectic volume of $M$. The main result of [20] states that for a suitable choice of an idempotent $a \in Q H_{\mathrm{ev}}(M)$, the function $\mu: \widetilde{\operatorname{Ham}}(M) \rightarrow \mathbb{R}$ given by

$$
\mu(\phi):=-\operatorname{vol}(M) \cdot \lim _{k \rightarrow+\infty} c\left(a, \phi^{k}\right) / k
$$

is a homogeneous quasi-morphism on the group $\widetilde{\operatorname{Ham}}(M)$ with a number of additional properties. More precisely, the following holds:

Quasi-additivity. There exists $K>0$, which depends only on $\mu$, so that

$$
|\mu(\phi \psi)-\mu(\phi)-\mu(\psi)| \leq K \quad \text { for all elements } \phi, \psi \in \widetilde{\operatorname{Ham}}(M) .
$$

Homogeneity. $\mu\left(\phi^{m}\right)=m \mu(\phi)$ for each $\phi$ and each $m \in \mathbb{Z}$.

To proceed with properties of $\mu$ we need the following notations. For a (timedependent) Hamiltonian $H$ on $M$ write $\phi_{H}$ for the element of $\widetilde{\operatorname{Ham}}(M)$ represented the identity-based path in $\operatorname{Ham}(M)$ given by the [0, 1]-time Hamiltonian flow generated by $H$. For an open $U \subset M$ denote by $\widetilde{\operatorname{Ham}}(U) \subset \widetilde{\operatorname{Ham}}(M)$ the subgroup of elements 
generated by Hamiltonians $H(x, t)=H_{t}(x)$ with supp $H_{t} \subset U$ for all $t \in \mathbb{S}^{1}$. Denote by Cal: $\widetilde{\operatorname{Ham}}(U) \rightarrow \mathbb{R}$ the classical Calabi homomorphism: $\operatorname{Cal}\left(\phi_{H}\right):=$ $\int_{0}^{1} \int_{U} H_{t} \omega^{n} d t$, where supp $H_{t} \subset U$ for all $t$.

Calabi property. If $U \subset M$ is open and displaceable, then the restriction of $\mu$ on $\widetilde{\operatorname{Ham}}(U) \subseteq \widetilde{\operatorname{Ham}}(M)$ is the Calabi homomorphism Cal: $\widetilde{\operatorname{Ham}}(U) \rightarrow \mathbb{R}$.

Lipschitz property. $\left|\mu\left(\phi_{F}\right)-\mu\left(\phi_{H}\right)\right| \leq \operatorname{vol}(M) \cdot\|F-H\|_{C^{0}}$.

Define now $\zeta: C^{\infty}(M) \rightarrow \mathbb{R}$ by

$$
\zeta(F)=\frac{\int_{M} F \omega^{n}}{\operatorname{vol}(M)}-\frac{\mu\left(\phi_{F}\right)}{\operatorname{vol}(M)}=\lim _{k \rightarrow+\infty} \frac{\bar{c}(a, k F)}{k} .
$$

Using the Lipschitz property of $\mu$, we readily extend $\zeta$ to a functional on $C(M)$. Let us check that $\zeta$ satisfies the axioms of a symplectic quasi-state. Since $\mu(\mathbf{1})=0$ in view of homogeneity of $\mu$, we get the normalization axiom. Invariance and monotonicity of spectral invariants yield the invariance and the monotonicity axioms respectively. The Calabi property of $\mu$ yields the vanishing axiom. To check the strong quasiadditivity axiom, note that if $\{F, G\}=0$ the diffeomorphisms $\phi_{F}$ and $\phi_{G}$ commute and $\phi_{F+G}=\phi_{F} \phi_{G}$. The desired result follows from the following general fact (which is an easy exercise): restriction of a homogeneous quasi-morphism to any abelian subgroup is a homomorphism. This completes the proof of Theorem 3.1.

\section{A partial symplectic quasi-state}

Let $M$ be a closed strongly semi-positive and rational symplectic manifold. For an element $\phi \in \widetilde{\operatorname{Ham}}(M)$ write for brevity $c(\phi)=c([M], \phi)$ and, as above, define $\mu$ as a homogenization of $c([M], \cdot)$ :

$$
\mu(\phi):=-\operatorname{vol}(M) \cdot \lim _{k \rightarrow+\infty} c\left(\phi^{k}\right) / k .
$$

It is easy to see that $\mu$ is not a quasi-morphism already when $M$ is the 2-torus - see the discussion following Question 8.7 in Section 8. Moreover, a similar argument actually shows that for any (strongly semi-positive, rational) symplectic direct product $M \times \mathbb{T}^{2 n}$ the homogenization of any spectral number $c(a, \cdot)$ cannot be a quasi-morphism.

In spite of this, $\mu$ has a number of nice properties which will enable us to show that the functional $\zeta$ given by (4) is a partial symplectic quasi-state. We shall need the following definition. Given a displaceable open set $U \subset M$, each $\phi \in \widetilde{\operatorname{Ham}}(M)$ can be represented as a product of elements of the form $\psi \theta \psi^{-1}$ with $\theta \in \widetilde{\operatorname{Ham}}(U)$. This follows from Banyaga's fragmentation lemma [9]. Denote by $\|\phi\|_{U}$ the minimal number of factors in such a product. 
Theorem 7.1. Suppose $M$ is strongly semi-positive and rational. The functional $\mu: \widetilde{\operatorname{Ham}}(M) \rightarrow \mathbb{R}$, given by (5), is well defined and has the following properties:

Controlled quasi-additivity. Given a displaceable open subset $U$ of $M$, there exists a constant $K$, depending only on $U$, so that

$$
|\mu(\phi \psi)-\mu(\phi)-\mu(\psi)| \leq K \min \left\{\|\phi\|_{U},\|\psi\|_{U}\right\}
$$

for any $\phi, \psi \in \widetilde{\operatorname{Ham}}(M)$.

Semi-homogeneity. $\mu\left(\phi^{m}\right)=m \mu(\phi)$ for any $\phi$ and any $m \in \mathbb{Z}_{\geq 0}$.

In addition it has the Calabi and Lipschitz properties defined in the previous section.

Postponing the proof, we first prove Theorem 4.1.

Proof of Theorem 4.1 (assuming Theorem 7.1). Define a functional $\zeta: C^{\infty}(M) \rightarrow$ $\mathbb{R}$ by formula (4). We claim that $\zeta$ is a partial symplectic quasi-state. Arguing exactly as in the end of the previous section, we check the monotonicity, vanishing, normalization and invariance axioms. Semi-homogeneity of $\mu$ yields that $\zeta(\lambda F)=$ $\lambda \zeta(F)$ for $\lambda \in \mathbb{N}$ and all smooth $F$. As a logical consequence we get that the same holds for all positive rational $\lambda$. Using the Lipschitz property of $\mu$, we pass to the limit and get this for all positive $\lambda$, thus establishing the semi-homogeneity axiom.

It remains to verify the partial additivity axiom. Assume that $\{F, H\}=0$ and supp $H$ is contained in a displaceable open subset $U$. Note that $\left\|\phi_{H}^{k}\right\|_{U}=1$ for all $k \in \mathbb{N}$. Since $\phi_{F}$ and $\phi_{H}$ commute we have (using controlled quasi-additivity of $\mu$ )

$$
\mu\left(\phi_{F} \phi_{H}\right)=\frac{1}{k} \mu\left(\left(\phi_{F} \phi_{H}\right)^{k}\right)=\frac{1}{k}\left(k \mu\left(\phi_{F}\right)+k \mu\left(\phi_{H}\right)+r_{k}\right),
$$

where $\left|r_{k}\right| \leq K$. Taking the limit as $k \rightarrow+\infty$ we get that

$$
\mu\left(\phi_{F} \phi_{H}\right)=\mu\left(\phi_{F}\right)+\mu\left(\phi_{H}\right)=\mu\left(\phi_{F}\right)+\int_{M} H \omega^{n},
$$

where the last equality follows from the Calabi property and the fact that supp $H$ is displaceable. Further, $\phi_{F+H}=\phi_{F} \phi_{H}$ since $F$ and $H$ commute. Substituting this into the definition of $\zeta$, we get $\zeta(F+H)=\zeta(F)$, as required. This completes the proof.

Proof of Theorem 7.1. The proof is divided into a sequence of lemmas. In what follows we fix an open displaceable subset $U$ of $M$ and write for simplicity $\|\phi\|:=\|\phi\|_{U}$. 
Lemma 7.2. There exists a constant $C \geq 0$ such that for any $\phi \in \widetilde{\operatorname{Ham}}(U)$

$$
0 \leq c(\phi)+c\left(\phi^{-1}\right) \leq C .
$$

Proof. Suppose $f \in \widetilde{\operatorname{Ham}}(M)$ is a lift of a Hamiltonian diffeomorphism displacing $U$. Then the "shift of the spectrum" trick of Y. Ostrover [38] (cf. [19], [20]) yields that for a certain $E \in \mathbb{R}$, depending on $\phi$,

$$
\begin{gathered}
c(f \phi)=c(f)+E, \\
c\left(f \phi^{-1}\right)=c(f)-E .
\end{gathered}
$$

Here we use the spectrality and the Lipschitz property of $c$. The signs in the formulae above comply with the sign convention as in [20]. Thus

$$
c(f \phi)+c\left(f \phi^{-1}\right)=2 c(f) .
$$

In view of the triangle inequality ${ }^{3}$,

$$
\begin{aligned}
0 & \leq c(\phi)+c\left(\phi^{-1}\right), \\
c(\phi) & \leq c(f \phi)+c\left(f^{-1}\right), \\
c\left(\phi^{-1}\right) & \leq c\left(f \phi^{-1}\right)+c\left(f^{-1}\right) .
\end{aligned}
$$

Hence

$$
0 \leq c(\phi)+c\left(\phi^{-1}\right) \leq c(f \phi)+c\left(f \phi^{-1}\right)+2 c\left(f^{-1}\right) \leq 2 c(f)+2 c\left(f^{-1}\right) .
$$

Set $C:=2 c(f)+2 c\left(f^{-1}\right)$. This is a non-negative number because of the triangle inequality. The lemma is proved.

Lemma 7.3. For any $\phi \in \widetilde{\operatorname{Ham}}(U)$ and any $\psi \in \widetilde{\operatorname{Ham}}(M)$ one has

$$
c(\phi)+c(\psi)-C \leq c(\phi \psi) \leq c(\phi)+c(\psi),
$$

where $C$ is the constant from the previous lemma.

Proof. The second inequality is just the triangle inequality. To obtain the first one, observe that the triangle inequality yields

$$
c(\psi) \leq c(\phi \psi)+c\left(\phi^{-1}\right) .
$$

\footnotetext{
${ }^{3}$ Note that, since $[M]$ is the unit in $Q H_{*}(M)$, the triangle inequality for $c(\cdot)=c([M], \cdot)$ has the form $c(\phi \psi) \leq c(\phi)+c(\psi)$.
} 
This, along with the previous lemma, implies

$$
c(\phi \psi) \geq c(\psi)-c\left(\phi^{-1}\right) \geq c(\psi)+c(\phi)-C .
$$

Using a straightforward inductive argument one generalizes the lemma above as follows. Take any $\phi_{1}, \ldots, \phi_{m}, \psi \in \widetilde{\operatorname{Ham}}(M)$ with $\left\|\phi_{i}\right\|=1$ for all $i$. Then

$$
\left|c\left(\phi_{1} \ldots \phi_{m} \psi\right)-\sum_{i=1}^{m} c\left(\phi_{i}\right)-c(\psi)\right| \leq m C .
$$

This formula (with $\psi=\mathbf{1}$ ) yields

$$
\left|c\left(\left(\phi_{1} \ldots \phi_{m}\right)^{l}\right)-l \sum_{i=1}^{m} c\left(\phi_{i}\right)\right| \leq \operatorname{lm} C .
$$

Take any $\phi \in \operatorname{Ham}(M)$ and represent it as $\phi=\phi_{1} \ldots \phi_{m}$ with $\left\|\phi_{i}\right\|=1$ for all $i$. Formula (7) implies that for some large enough positive $E$ (depending on $\phi$ ) the sequence $\left\{c\left(\phi^{l}\right)+E l\right\}_{l \in \mathbb{N}}$ is non-negative. On the other hand, because of the triangle inequality, this sequence is sub-additive. This yields the existence and finiteness of $\lim _{l \rightarrow+\infty}\left(c\left(\phi^{l}\right)+E l\right) / l$ and, accordingly, of $\lim _{l \rightarrow+\infty} c\left(\phi^{l}\right) / l$. Therefore the function $\mu$ is well defined. The semi-homogeneity of $\mu$ follows immediately from its definition. The proof of the Lipschitz property of $\mu$ simply repeats the proof of a similar Proposition 3.5 in [20].

Now we are going to check controlled quasi-additivity of $\mu$. Assume without loss of generality that the volume of $M$ equals 1 , so that

$$
\mu(\phi)=-\lim _{k \rightarrow+\infty} c\left(\phi^{k}\right) / k
$$

We claim that for $\phi, \psi \neq \mathbf{1}$,

$$
|\mu(\phi \psi)-\mu(\phi)-\mu(\psi)| \leq 2 C \cdot \min (2\|\phi\|-1,2\|\psi\|-1) .
$$

The controlled quasi-additivity follows immediately from (8) if one sets $K:=4 C$. We prove the claim by induction on $m:=\min (\|\phi\|,\|\psi\|)$.

Induction basis $m=1$. Assume without loss of generality that $\|\phi\|=1$. Note that

$$
(\phi \psi)^{k}=\left(\prod_{i=0}^{i=k-1} \psi^{i} \phi \psi^{-i}\right) \cdot \psi^{k} .
$$

Applying (6) and using the conjugation invariance of $c(\cdot)$ we get

$$
\left|c\left((\phi \psi)^{k}\right)-k c(\phi)-c\left(\psi^{k}\right)\right| \leq C k .
$$


Combining this with inequality

$$
\left|c\left(\phi^{k}\right)-k c(\phi)\right| \leq C k,
$$

which follows from (7), dividing by $k$ and passing to the limit as $k \rightarrow+\infty$ we get the desired result.

Induction step $m \mapsto m+1$. Assume without loss of generality that $\|\phi\|=m+1$. Then $\phi$ can be decomposed as $\phi=\phi_{m} \phi_{1}$ where $\left\|\phi_{m}\right\|=m$ and $\left\|\phi_{1}\right\|=1$. Using the induction assumption we have

$$
\begin{gathered}
\left|\mu\left(\phi_{m} \phi_{1} \psi\right)-\mu\left(\phi_{m}\right)-\mu\left(\phi_{1} \psi\right)\right| \leq 2 C(2 m-1), \\
\left|\mu\left(\phi_{1} \psi\right)-\mu\left(\phi_{1}\right)-\mu(\psi)\right| \leq 2 C
\end{gathered}
$$

and

$$
\left|\mu\left(\phi_{1}\right)+\mu\left(\phi_{m}\right)-\mu\left(\phi_{m} \phi_{1}\right)\right| \leq 2 C .
$$

Adding up these inequalities we get that

$$
|\mu(\phi \psi)-\mu(\phi)-\mu(\psi)| \leq 2 C(2 m+1),
$$

as desired. This completes the proof of the claim and of the controlled quasi-additivity.

Finally, the proof of the Calabi property of $\mu$ virtually repeats the proof of a similar Proposition 3.3 in [20]. The symplectic invariance of $\mu$ follows from the symplectic invariance of the spectral numbers. This finishes the proof of Theorem 7.1.

\section{Symplectic quasi-states on surfaces}

Symplectic quasi-measures. A quasi-measure on a symplectic manifold $M$ is called symplectic if it is $\operatorname{Symp}_{0}(M)$-invariant and vanishes on displaceable closed subsets.

Here we discuss this notion in the case when $M$ is a closed surface equipped with an area form. According to the general construction from [3], any quasi-measure $\tau$ gives rise to a quasi-state $\zeta_{\tau}$. Roughly speaking, the definition of $\zeta_{\tau}$ is as follows. For a function $F \in C(M)$ define a measure $\sigma_{F}$ on $\mathbb{R}$ by its values on intervals

$$
\sigma_{F}([a ; b)):=\tau(\{F \geq a\})-\tau(\{F \geq b\},
$$

and put $\zeta_{\tau}(F):=\int_{\mathbb{R}} s \cdot d \sigma_{F}(s)$. If $\tau$ is a symplectic quasi-measure, the quasi-state $\zeta_{\tau}$ automatically satisfies all the axioms of a symplectic quasi-state except, possibly, strong quasi-additivity stating that $\zeta_{\tau}$ is linear on the centralizer (with respect to the Poisson bracket) of any smooth function $F$. 
Theorem 8.1. On a closed surface, the strong quasi-additivity axiom follows from the usual quasi-linearity. In particular, any symplectic quasi-measure gives rise to a symplectic quasi-state.

Proof. Let $F, G$ be a pair of $C^{\infty}$-smooth functions on a closed surface $M$ with $\{F, G\}=0$. The Poisson-commutativity can be interpreted as follows: the differential of the map

$$
\Phi: M \rightarrow \mathbb{R}^{2}, x \mapsto(F(x), G(x)),
$$

has rank $\leq 1$ for at each point $x \in M$. Put $\Delta:=\operatorname{Image}(\Phi)$. Denote by $d_{c}$ and $d_{h}$ the covering dimension and the Hausdorff dimension of $\Delta$ respectively. It is a standard fact of dimension theory that $d_{c} \leq d_{h}$, see e.g. the proof of Theorem (6.2.10) in Edgar's book [17]. Further, $d_{h} \leq 1$. This follows from a result of Dubovickii [16] which is a partial case of a more general theorem of Sard [40]. Therefore $d_{c} \leq 1$.

Define a quasi-state $\eta$ on $C(\Delta)$ by $\eta(H):=\zeta_{\tau}\left(\Phi^{*} H\right)$. The Wheeler-Shakhmatov Theorem [26], [44] implies that every quasi-state on a normal topological space (and hence on any metric space) of covering dimension $\leq 1$ is linear. Hence $\eta$ is linear. Applying this result to the restriction of the coordinate functions on $\mathbb{R}^{2}$ to $\Delta$ we get that

$$
\zeta_{\tau}(F+G)=\zeta_{\tau}(F)+\zeta_{\tau}(G)
$$

as required.

Note that in the proof above we used that the functions $F$ and $G$ are infinitely smooth in order to deduce inequality $d_{h} \leq 1$ from the Dubovickii-Sard theorem.

Problem 8.2. Extend identity (9) to Poisson-commuting functions of finite smoothness.

For instance, one can try to find a uniform approximation of the pair $(F, G)$ by a Poisson-commuting pair of $C^{\infty}$-functions.

Remark 8.3. In contrast to the case of surfaces, the only known to us example of a symplectic quasi-measure on higher-dimensional manifolds comes from the "Floerhomological" symplectic quasi-state whose existence is established in Theorem 3.1.

Remark 8.4. In the case $\operatorname{dim} M>2 \mathrm{D}$. Grubb [25] constructed examples of quasistates which are not strongly quasi-additive. The quasi-measures in the examples of Grubb do not necessarily vanish on displaceable sets (and hence are not symplectic) but may vanish on a stem. The push-forward of such a quasi-measure by a moment map of a finite-dimensional Poisson-commutative subspace of $C^{\infty}(M)$ is not necessarily a measure. 
Now we address a question about existence and uniqueness of symplectic quasistates and quasi-measures on surfaces.

The 2-sphere. The group $\operatorname{Ham}\left(\mathbb{S}^{2}\right)$ admits a Calabi quasi-morphism [20], which in accordance with our discussion in Section 6 yields existence of a symplectic quasistate and a symplectic quasi-measure on $C\left(\mathbb{S}^{2}\right)$. Theorem 5.2 in [20] shows that any two Calabi quasi-morphisms on $\operatorname{Ham}\left(\mathbb{S}^{2}\right)$ coincide on the set of elements generated by time-independent Hamiltonians. The same argument proves that any two symplectic quasi-states coincide on the set of smooth Morse functions on $\mathbb{S}^{2}$. Hence $C\left(\mathbb{S}^{2}\right)$ carries unique symplectic quasi-state and quasi-measure.

An explicit calculation presented in [20] shows that the restriction of this symplectic quasi-state, say $\zeta$, to the subalgebra $\mathscr{A}_{F} \subset C(M)$ generated by a single Morse function $F \in C^{\infty}\left(\mathbb{S}^{2}\right)$ is multiplicative: $\zeta(G H)=\zeta(G) \zeta(H)$ for all $G, H \in \mathcal{A}_{F}$. Using this along with the continuity of $\zeta$ one can easily show that $\zeta$ is multiplicative on $\mathcal{A}_{F}$ for any $F \in C\left(\mathbb{S}^{2}\right)$. Now a theorem of Aarnes [4] yields that the corresponding quasi-measure is simple: it takes values 0 and 1 only. It is unclear whether this phenomenon persists in higher dimensions, thus we pose the next question.

Question 8.5. Consider the "Floer-homological" symplectic quasi-state $\zeta$ on the complex projective space $\mathbb{C P}^{n}$ constructed in Theorem 3.1. Is it multiplicative when $n \geq 2$ ? In particular, is it true that $\zeta\left(F^{2}\right)=\zeta(F)^{2}$ for all continuous functions $F$ on $\mathbb{C P}^{n}$ ?

For completeness, we present the formula for $\zeta$ on $\mathcal{A}_{F}$, where $F$ is a Morse function, obtained in [20]. Assume that the total area of the sphere equals 1. One shows that there exists unique (may be, singular) connected component of a level set of $F$, say $\gamma$, so that the area of any connected component of $\mathbb{S}^{2} \backslash \gamma$ is $\leq \frac{1}{2}$. Note that every $G \in \mathcal{A}_{F}$ is constant on connected components of level sets of $F$. It turns out that

$$
\zeta(G)=G(\gamma) .
$$

A symplectic quasi-measure $\tau$ corresponding to $\zeta$ can be described as follows (we thank D. Grubb who pointed this out to us). A set $A \subset \mathbb{S}^{2}$ is called solid if both $A$ and $\mathbb{S}^{2} \backslash A$ are connected. According to the results of Aarnes [5] and Aarnes and Rustad [6], the quasi-measure $\tau$ is completely defined by the following condition: for a closed solid set $A \subset \mathbb{S}^{2}$ one has $\tau(A)=1$ if the Lebesgue measure of $A$ is greater or equal to $1 / 2$ and $\tau(A)=0$ otherwise.

The 2-torus. Existence of a symplectic quasi-measure, say $\tau$, in this case follows from a work of Grubb (see Theorem 32 of [24], where the auxiliary quasi-measures used in the definition of $\tau$ are taken to be the standard Lebesgue measure). The value of $\tau$ on any 2-dimensional smooth connected closed submanifold with boundary $W \subset \mathbb{T}^{2}$ can be calculated as follows (see Theorem 32 of [24]). If $W$ is contractible 
in $\mathbb{T}^{2}$ we have $\tau(W)=0$. If $W$ is non-contractible and $\partial W$ has $k \geq 0$ contractible connected components that bound pair-wise disjoint discs $D_{1}, \ldots, D_{k}$ (in case $k=0$ there are no discs), then

$$
\tau(W)=\operatorname{Area}(W)+\sum_{i=1}^{k} \operatorname{Area}\left(D_{i}\right) .
$$

Remark 8.6. It would be interesting to describe all symplectic quasi-measures on the 2-torus; for more examples of such quasi-measures see a recent preprint [28] by Knudsen.

By Theorem 8.1 above, a symplectic quasi-measure on $\mathbb{T}^{2}$ gives rise to a symplectic quasi-state.

Question 8.7. Is Grubb's symplectic quasi-measure associated to a quasi-morphism on $\operatorname{Ham}\left(\mathbb{T}^{2}\right)$ ?

Such a quasi-morphism, if exists, cannot come from spectral numbers described in Section 5. To see this denote by $\tau$ any symplectic quasi-measure on $\mathbb{T}^{2}$. Introduce coordinates $(p, q) \bmod 1$ on $\mathbb{T}^{2}$ so that the symplectic form is given by $d p \wedge d q$. Let $\alpha=\{p=0\}$ and $\beta=\{p=1 / 2\}$ be two meridians dividing the torus into two open annuli $A=\{p \in(0 ; 1 / 2)\}$ and $B=\{p \in(1 / 2 ; 1)\}$ of equal area. Note that

$$
\tau(A)+\tau(B)+\tau(\alpha)+\tau(\beta)=1 .
$$

The Symp ${ }_{0}$-invariance of $\tau$ yields $\tau(A)=\tau(B)$ as well as $\tau(\alpha)=\tau(\beta)=0$ (the torus contains an arbitrarily large number of pair-wise disjoint symplectic shifts of a meridian). Thus, putting $A^{\prime}=A \cup \alpha \cup \beta$, we have $\tau\left(A^{\prime}\right)=1 / 2$. On the other hand, choose a sequence of cut-off functions $F_{i}(p)$ approximating the characteristic function of $A^{\prime}$ so that the only critical values of $F_{i}$ are 0 and 1 . The key feature of the Hamiltonian flow generated by $F_{i}$ is that its only contractible closed orbits are the critical points, hence the action spectrum $\overline{\operatorname{spec}}\left(t F_{i}\right)$ equals $\{0 ; t\}$. Hence, using continuous dependence of spectral numbers on the Hamiltonian, we get that for every homology class $a \in H_{*}\left(\mathbb{T}^{2}\right)$, we have either $\bar{c}\left(a, t F_{i}\right)=0$ or $\bar{c}\left(a, t F_{i}\right)=t$. Substituting this into the right term of formula (4), we get that $\zeta\left(F_{i}\right)$, if well defined, must be either 0 or 1 and hence $\tau\left(A^{\prime}\right) \neq 1 / 2$. This contradiction proves the claim.

Note that Hamiltonians $F_{i}$ above have a wealth of non-contractible periodic orbits. In principle, the symplectic field theory [18], or, more precisely, its version called branched Floer homology (work in progress by V. Ginzburg and E. Kerman) which deals with Hamiltonian diffeomorphisms, may lead to a generalization of spectral numbers which takes into account non-contractible orbits as well. It would be interesting to understand whether this path leads to a symplectic quasi-measure. 


\section{Digging out a stem}

Assume that one faces the problem of the following type: "Prove that a certain specific Lagrangian submanifold $L$ of a symplectic manifold $M$ is non-displaceable". The mainstream approach to this problem is to show that the Lagrangian Floer homology of $L$ is well defined and does not vanish. Our results above give rise to another potential approach (cf. [13]): show that $L$ is a stem (see Definition 2.3) and deduce the non-displaceability of $L$ from Theorem 2.1. Let us emphasize that this approach is not "soft": unveiling the proof, one sees that we use an information about the asymptotic behaviour of Hamiltonian Floer homology for Hamiltonians concentrated near $L$. While in certain situations our method is simpler, it does not provide a lower bound on the number of intersections (assuming they are all transversal) between $L$ and its image under a Hamiltonian isotopy - a bound which is usually given by the Lagrangian Floer homology approach whenever it works.

Below we illustrate our approach for the Lagrangian Clifford torus in $\mathbb{C P}^{n}$ and for a similar torus in a monotone blow-up of $\mathbb{C P}^{2}$ at one point.

The Clifford torus in $\mathbb{C} \mathbf{P}^{n}$. This example is taken from [13]. Let $M$ be $\mathbb{C P}^{n}$ with the Fubini-Study symplectic form. Consider the standard Hamiltonian $\mathbb{T}^{n}$-action on $M$ whose moment polytope is a simplex in $\mathbb{R}^{n}$. Denote by $L$ the Lagrangian torus which is the fiber of the moment map over the barycenter of the simplex - it is called the Clifford torus and can be described as

$$
L:=\left\{\left[z_{0}: \cdots: z_{n}\right] \in \mathbb{C P}^{n}|| z_{0}|=\cdots=| z_{n} \mid\right\} .
$$

All the fibers of the moment map, other than $L$, are displaceable - this easily follows from the observation that permutations of homogeneous coordinates can be realized by Hamiltonian diffeomorphisms of $\mathbb{C P}^{n}$ coming from the natural action of $\mathrm{PU}(n+1)$ on $\mathbb{C P}^{n}$. Thus $L$ is a stem. Hence, according to Corollary $2.2, L$ is non-displaceable [13].

In fact, the non-displaceability of $L$ can be also proved by means of the Lagrangian Floer homology. In this way Cho [14] showed that if an image of $L$ under a Hamiltonian isotopy is transversal to $L$ then the number of intersections between them must be at least $2^{n}$ (which is the sum of the Betti numbers of $L$ ).

The Clifford torus in the monotone blow-up of $\mathbb{C} P^{2}$ at one point. Our interest in this example is due to the fact that in this case the obstructions to displaceability of $L$ coming from the Lagrangian Floer homology do vanish according to a result by Cho and $\mathrm{Oh}[15]$.

Here is the description of $L$. Consider a spherical shell $W$ lying in the standard symplectic linear space $\mathbb{C}^{2}$ :

$$
W=\left\{\left(u_{1}, u_{2}\right) \in \mathbb{C}^{2} \mid \frac{1}{3} \leq \pi\left(\left|u_{1}\right|^{2}+\left|u_{2}\right|^{2}\right) \leq 1\right\} .
$$


Making a symplectic cut (i.e. collapsing the boundaries along the fibers of the characteristic foliation) we get a closed symplectic 4-manifold $M$ which is one of the models of the blow up of $\mathbb{C P}^{2}$ at one point. The details of the construction can be extracted from the description of the symplectic structure on $M$ given in [7], p. 61. The symplectic manifold $M$ is spherically monotone. A Lagrangian torus

$$
L:=\left\{\pi\left|u_{1}\right|^{2}=\pi\left|u_{2}\right|^{2}=\frac{1}{3}\right\}
$$

is called the Clifford torus of $M-$ it can be viewed as the Clifford torus in $\mathbb{C P}^{2}$ which "survived" the blow-up.

Theorem 9.1. The Clifford torus $L \subset M$ is a stem.

Combining this with Corollary 2.2 we get that $L$ is non-displaceable.

Proof. Consider a Hamiltonian action of the 2-torus on $M$, which in the spherical shell model is defined by its moment map

$$
\Phi: W \rightarrow \mathbb{R}^{2}, \quad\left(u_{1}, u_{2}\right) \mapsto\left(\pi\left|u_{1}\right|^{2}, \pi\left|u_{2}\right|^{2}\right) .
$$

We shall show that $L$ is the stem of a Poisson-commutative subspace generated by the coordinate functions of $\Phi$. The image $\triangle$ of $\Phi$ is a trapezoid $A B C D$ in the plane with the vertices

$$
A=(0,1 / 3), \quad B=(1 / 3,0), \quad C=(1,0), D=(0,1) .
$$

The Clifford torus $L$ is given by $\Phi^{-1}(Q)$, where $Q=(1 / 3,1 / 3)$.

Claim. The fiber $\Phi^{-1}(X)$ is displaceable for every $X \neq Q$.

We use the following notation for lines and segments on the plane: $P R$ stands for the line passing through points $P$ and $R,[P R)$ denotes the segment with vertices $P$ and $R$ so that $P$ is included and $R$ is excluded and so on. We write $|P R|$ for the Euclidean length of $[P R]$.

Consider the points

$$
P=(1 / 6,1 / 6) \in[A B], \quad R=(1 / 2,1 / 2) \in[C D] .
$$

Case I: $X \notin[P R]$. The unitary transformation $S:\left(u_{1}, u_{2}\right) \rightarrow\left(u_{2}, u_{1}\right)$ of $W$ commutes with the $\mathbb{T}^{2}$-action and induces the symmetry of $\Delta$ over the line $P R$ which sends $X$ to a point $X^{\prime} \neq X$. Hence $S\left(\Phi^{-1}(X)\right) \cap \Phi^{-1}(X)=\emptyset$, which proves the claim in this case.

In order to proceed further, take the point $E=(2 / 3,1 / 3) \in[C D]$. The segment [AE] divides $\Delta$ into a triangle $\Delta^{\prime}$ and a parallelogram $\Pi$. We assume that $\Delta^{\prime}$ contains 
segments $[D A)$ and $[D E)$ and does not contain $[A E]$, while $\Pi$ contains $[B A)$ and $[B C)$ and does not contain the two other edges.

Case II: $X \in(Q R]$. The set $\Phi^{-1}\left(\Delta^{\prime}\right)$ is $\mathbb{T}^{2}$-equivariantly symplectomorphic to the standard symplectic ball $\left\{\pi\left(\left|w_{1}\right|^{2}+\left|w_{2}\right|^{2}\right)<2 / 3\right\}$ (the vertex $D$ corresponds to the center of the ball). This follows from the local version of Delzant theorem - see [27]. The unitary transformation $\left(w_{1}, w_{2}\right) \rightarrow\left(w_{2}, w_{1}\right)$ of this ball commutes with the $\mathbb{T}^{2}$-action and induces an affine involution of $\Delta^{\prime}$ whose fixed point set coincides with $[D Q)$. This involution sends $X$ to some point $X^{\prime} \neq X$. We conclude that the torus $\Phi^{-1}(X)$ can be sent to $\Phi^{-1}\left(X^{\prime}\right)$ by a Hamiltonian isotopy, and therefore is displaceable.

Case III: $X \in[P Q)$. The set $\Phi^{-1}(\Pi)$ is $\mathbb{T}^{2}$-equivariantly symplectomorphic to the standard symplectic polydisc $\mathcal{C}=\mathscr{D}_{1} \times \mathscr{D}_{2}$ with

$$
\mathscr{D}_{1}=\left\{\pi\left|w_{1}\right|^{2}<2 / 3\right\}, \quad \mathscr{D}_{2}=\left\{\pi\left|w_{2}\right|^{2}<1 / 3\right\}
$$

(the vertex $B$ corresponds to the center of the polydisc). This again follows from the local version of Delzant theorem [27]. The projection of $\mathcal{C}$ to $\mathcal{D}_{1}$ sends the torus $\Phi^{-1}(X)$ to a circle $\Gamma:=\left\{\pi\left|w_{1}\right|^{2}=r\right\}$ which encloses a disc of area $r$. The area $r$ corresponding to the point $X$ can be calculated as follows. Let $Y$ be the projection of $X$ to $B C$ along $A B$. Then

$$
\frac{r}{\operatorname{Area}\left(D_{1}\right)}=\frac{|B Y|}{|B C|}<\frac{1}{2} .
$$

This inequality guarantees that $\Gamma$ is displaceable in $\mathscr{D}_{1}$ by a Hamiltonian transformation of $D_{1}$. Lifting this transformation to $\mathcal{C}$ we get that $\Phi^{-1}(X)$ is displaceable. This completes the proof of the claim, and hence of the theorem.

Sometimes even in seemingly simple situations it is hard to decide whether a given Lagrangian submanifold is a stem. For instance, we do not know an answer to the following question:

Question 9.2. Consider $\mathbb{R} P^{2} \subset \mathbb{C P}$ or the anti-diagonal in the monotone $\mathbb{S}^{2} \times \mathbb{S}^{2}$. Are they stems?

\section{On the history and the physical meaning of quasi-states}

The notion of quasi-state has an amusing history. To discuss it let us recall the mathematical model of quantum mechanics which goes back to von Neumann's famous book [43] published in 1932: Its basic ingredients are the real Lie algebra of observables $\mathscr{A}_{q}$ ( $q$ for quantum) whose elements (in the simplest version of the theory) 
are hermitian operators on a finite-dimensional complex Hilbert space $H$ and the Lie bracket is given by

$$
[A, B]_{\hbar}=\frac{i}{\hbar}(A B-B A),
$$

where $\hbar$ is the Planck constant. Observables represent physical quantities such as energy, position, momentum etc. The state of a quantum system is given by a functional $\zeta: \mathcal{A}_{q} \rightarrow \mathbb{R}$ which satisfies the following axioms:

Additivity. $\zeta(A+B)=\zeta(A)+\zeta(B)$ for all $A, B \in \mathcal{A}_{q}$.

Homogeneity. $\zeta(c A)=c \zeta(A)$ for all $c \in \mathbb{R}$ and $A \in \mathcal{A}_{q}$.

Positivity. $\zeta(A) \geq 0$ provided $A \geq 0$.

Normalization. $\zeta(\mathbf{1})=1$.

As a consequence of these axioms von Neumann proved that for every quantum state $\zeta$ there exists a non-negative Hermitian operator $U_{\zeta}$ with trace 1 such that $\zeta(A)=\operatorname{tr}\left(U_{\zeta} A\right)$ for all $A \in \mathcal{A}_{q}$. An easy consequence of this formula is that for every state $\zeta$ there exists an observable $A$ such that

$$
\zeta\left(A^{2}\right)-\zeta(A)^{2}>0 .
$$

In his book von Neumann adopted a statistical interpretation of quantum mechanics according to which the value $\zeta(A)$ is considered as the expectation of a physical quantity represented by $A$ in the state $\zeta$. In this interpretation the equation (10) says that there are no dispersion-free states. This result led von Neumann to a conclusion which in the language of quantum mechanics can be formulated as the impossibility to introduce hidden variables into the quantum theory. This conclusion caused a (seemingly never ending) discussion among physicists which (citing Ballentine [8], p. 374) "was unfortunately clouded by emotionalism". A number of prominent physicists, including Bohm and Bell, disagreed with the additivity axiom of a quantum state. Their reasoning was that the formula $\zeta(A+B)=\zeta(A)+\zeta(B)$ makes sense a priori only if observables $A$ and $B$ are simultaneously measurable, that is commute: $[A, B]_{\hbar}=0$. We refer to Bell's paper [10] for an account of this discussion.

In 1957 Gleason [23] proved a remarkable rigidity-type theorem which can be considered as an additional argument in favor of von Neumann's additivity axiom. Recall that two hermitian operators on a finite-dimensional Hilbert space commute if and only if they can be written as polynomials of the same self-adjoint operator. Let us introduce a quasi-state on $\mathcal{A}_{q}$ as a real-valued functional which satisfies the homogeneity, positivity and normalization axioms above, while the additivity axiom is replaced by one of the two equivalent axioms:

Quasi-additivity-I. $\zeta(A+B)=\zeta(A)+\zeta(B)$ provided that $A$ and $B$ commute: $[A, B]_{\hbar}=0$. 
Quasi-additivity-II. $\zeta(A+B)=\zeta(A)+\zeta(B)$ provided that $A$ and $B$ belong to a single-generated subalgebra of $\mathcal{A}_{q}$.

According to the Gleason theorem, every quasi-state on $\mathcal{A}_{q}$ is linear (that is, a state) provided the complex dimension of the Hilbert space $H$ is at least 3 (it is an easy exercise to show that in the two-dimensional case there are plenty of non-linear quasi-states).

Let us turn now to the mathematical model of classical mechanics. Here the algebra $\mathcal{A}_{c}$ of observables ( $c$ for classical) is the space of continuous functions $C(M)$ on a symplectic manifold $M$. The Lie bracket is defined as the Poisson bracket on the dense subspace $C^{\infty}(M) \subset C(M)$. A natural question is whether the conclusion of the Gleason theorem remains valid in the classical context. We immediately face a dilemma: which of two definitions of quasi-additivity one should adopt as the starting point of such an extension. Adopting the second one, we arrive to the definition of a quasi-state given by Aarnes. It does not involve the symplectic structure and gives rise to the theory of quasi-states on general topological spaces. Adopting the first one, and taking into account the Correspondence Principle according to which the bracket $[,]_{\hbar}$ corresponds to the Poisson bracket $\{$,$\} in the classical limit \hbar \rightarrow 0$, we get a definition which involves the strong quasi-additivity axiom: $\zeta(F+G)=\zeta(F)+\zeta(G)$ whenever $\{F, G\}=0$, see Section 3. According to Theorem 8.1 above both definitions coincide in dimension 2. However, as it was mentioned in Remark 8.4, strong quasi-additivity is strictly stronger in higher dimensions. For the sake of brevity, we refer to non-linear strongly quasi-additive quasi-states on symplectic manifolds as to strong quasi-states.

In light of this discussion, Theorems 3.1 and 8.1 above which establish the existence of strong quasi-states on certain symplectic manifolds can be viewed as an "anti-Gleason phenomenon" in classical mechanics. This interpretation is far from being transparent. Let us indicate two points which require further clarification.

First, recall that the algebra $\mathcal{A}_{c}$ of classical observables can be considered as a suitable limit of matrix algebras $\mathcal{A}_{q}$ where the dimension $N$ of the underlying Hilbert space $H$ tends to $\infty$ and the Planck constant $\hbar$ tends to 0 . We refer the reader to Madore's paper [33] dealing with the case where the classical phase space is the 2-dimensional sphere. For certain symplectic manifolds the algebra $\mathcal{A}_{c}$ carries a strong quasi-state, say, $\zeta$. At the same time the Gleason theorem rules out existence of a non-linear quasi-state on $\mathcal{A}_{q}$ for every given values of $N$ and $\hbar$. It would be interesting to understand what is a footprint of $\zeta$ in the quantum world. For instance, do the algebras $\mathscr{A}_{q}$ carry a weaker object (a kind of "approximate quasi-state" still to be defined) which converges to $\zeta$ ?

Second, by the analogy with quantum mechanics, one can speculate that Poisson non-commuting functions $F$ and $G$ with $\zeta(F+G) \neq \zeta(F)+\zeta(G)$ are not simultaneously measurable. Does there exist an explanation of this phenomenon in terms of classical mechanics? An extra difficulty here is due to the fact that some strong quasi-states are dispersion-free. Therefore one cannot refer to uncertainty as to the 
reason for the lack of simultaneous measurability.

Acknowledgements. We are grateful to P. Biran for his generous help with this paper. We also thank D. Grubb, Y. Karshon, Y. Ostrover, M. Symington and Y. Yomdin for useful discussions, and the anonymous referee for corrections.

\section{References}

[1] J. F. Aarnes, Physical states on a $C^{*}$-algebra. Acta Math. 122 (1969), 161-172. Zbl 0183.14203 MR 0247482

[2] J. F. Aarnes, Quasi-states on $C^{*}$-algebras. Trans. Amer. Math. Soc. 149 (1970), 601-625. Zbl 0212.15403 MR 0282602

[3] J. F. Aarnes, Quasi-states and quasi-measures. Adv. Math. 86 (1991), 41-67. Zbl 0744.46052 MR 1097027

[4] J. F. Aarnes, Pure quasi-states and extremal quasi-measures. Math. Ann. 295 (1993), 575-588. Zbl 0791.46028 MR 1214949

[5] J. F. Aarnes, Construction of non-subadditive measures and discretization of Borel measures. Fund. Math. 147 (1995), 213-237. Zbl 0842.28004 MR 1348720

[6] J. F. Aarnes, A. B. Rustad, Probability and quasi-measures - a new interpretation. Math. Scand. 85 (1999), 278-284. Zbl 0967.28014 MR 1724240

[7] M.Audin, Symplectic and almost complex manifolds. In Holomorphic curves in symplectic geometry, Progr. Math. 117, Birkhäuser, Basel 1994, , 41-74. MR 1274926

[8] L. E. Ballentine, The statistical interpretation of quantum mechanics. Rev. Modern Phys. 42 (1970), 358-381. Zbl 0203.27801

[9] A. Banyaga, Sur la structure du groupe des difféomorphismes qui préservent une forme symplectique. Comment. Math. Helv. 53 (1978), 174-227. Zbl 0393.58007 MR 0490874

[10] J. S. Bell, On the problem of hidden variables in quantum mechanics. Rev. Modern Phys. 38 (1966), 447-452. Zbl 0152.23605 MR 0208927

[11] P. Biran, Geometry of symplectic intersections. In Proceedings of the International Congress of Mathematicians, Vol. II, Higher Education Press, Beijing 2002, 241-255. Zbl 1021.53058 MR 1957037

[12] P. Biran, K. Cieliebak, Symplectic topology on subcritical manifolds. Comment. Math. Helv. 76 (2001), 712-753. Zbl 1001.53057 MR 1881704

[13] P. Biran, M. Entov, L. Polterovich, Calabi quasimorphisms for the symplectic ball. Commun. Contemp. Math. 6 (2004), 793-802. Zbl 02165920 MR 2100764

[14] C.-H. Cho, Holomorphic disc, spin structures and Floer cohomology of the Clifford torus. Int. Math. Res. Not. 35 (2004), 1803-1843. MR 2057871

[15] C.-H. Cho, Y.-G. Oh, Floer cohomology and disc instantons of Lagrangian torus fibers in Fano toric manifolds. Preprint, math.SG/0308225, 2003. 
[16] A. Ya. Dubovickii, On differentiable mappings of an $n$-dimensional cube into a $k$ dimensional cube (in Russian). Mat. Sb. N.S. 32 (74) (1953), 443-464. Zbl 0050.28102 MR 0057964

[17] G. A. Edgar, Measure, Topology and Fractal Geometry. Undergrad. Texts Math., SpringerVerlag, New York 1990. Zbl 0727.28003 MR 1065392

[18] Y. Eliashberg, A. Givental, H. Hofer, Introduction to symplectic field theory. Geom. Funct. Anal. 2000, Special Volume, Part II, 560-673. Zbl 0989.81114 MR 1826267

[19] M. Entov, Commutator length of symplectomorphisms. Comment. Math. Helv. 79 (2004), 58-104. Zbl 1048.53056 MR 2031300

[20] M. Entov, L. Polterovich, Calabi quasimorphism and quantum homology. Internat. Math. Res. Notices 30 (2003), 1635-1676. Zbl 1047.53055 MR 1979584

[21] K. Fukaya, K. Ono, Arnold conjecture and Gromov-Witten invariant. Topology 38 (1999), 933-1048. Zbl 0946.53047 MR 1688434

[22] I. Gelfand, N. Vilenkin, Generalized functions. Vol. 4: Applications of harmonic analysis. Academic Press, New York, London 1964. Zbl 0136.11201 MR 0435834

[23] A. M. Gleason, Measures on the closed subspaces of a Hilbert space. J. Math. Mech. 6 (1957), 885-893. Zbl 0078.28803 MR 0096113

[24] D. J. Grubb, Irreducible partitions and the construction of quasi-measures. Trans. Amer. Math. Soc. 353 (2001), 2059-2072. Zbl 0968.28007 MR 1813607

[25] D. J. Grubb, Private communication. November 2004.

[26] D. J. Grubb, T. LaBerge, Spaces of quasi-measures. Canad. Math. Bull. 42 (1999), 291-297. Zbl 0938.28009 MR 1703689

[27] Y. Karshon, Appendix to the paper "Symplectic packings and algebraic geometry" by D. McDuff and L. Polterovich. Invent. Math. 115 (1994), 431-434. Zbl 0833.53029 MR 1262938

[28] F. Knudsen, New topological measures on the torus. Fund. Math. 185 (2005), 287-293.

[29] D. Kotschick, What is . . a quasi-morphism? Notices Amer. Math. Soc. 51 (2004), 208-209. Zbl 02115064 MR 2026941

[30] G. Liu, G. Tian, Floer homology and Arnold conjecture. J. Differential Geom. 49 (1998), 1-74. Zbl 0917.58009 MR 1642105

[31] G. Liu, G. Tian, On the equivalence of multiplicative structures in Floer homology and quantum homology. Acta Math. Sin. (Engl. Ser.) 15 (1999), 53-80. Zbl 0928.53041 MR 1701133

[32] G. Lu, An explicit isomorphism between Floer homology and quantum homology. Pacific J. Math. 213 (2004), 319-363. Zbl 02113419 MR 2036923

[33] J. Madore, The fuzzy sphere. Classical Quantum Gravity 9(1992), 69-87. Zbl 0742.53039 MR 1146044

[34] D. McDuff, D. Salamon, J-holomorphic curves and symplectic topology. Amer. Math. Soc. Colloq. Publ. 52, Amer. Math. Soc., Providence, RI, 2004. Zbl 02121182 MR 2045629

[35] R. Narasimhan, Analysis on real and complex manifolds. Adv. Stud. Pure Math. 1, Masson \& Cie, Éditeurs, Paris; North-Holland Publishing Co., Amsterdam 1968. Zbl 0188.25803 MR 0251745 
[36] Y.-G. Oh, Construction of spectral invariants of Hamiltonian diffeomorphisms on general symplectic manifolds. In The breadth of symplectic and Poisson geometry, Progr. Math. 232, Birkhäuser, Boston 2005, 525-570. MR 2103018

[37] Y.-G. Oh, Floer mini-max theory, the Cerf diagram, and the spectral invariants. Preprint, math.SG/0406449, 2004.

[38] Y. Ostrover, A comparison of Hofer's metrics on Hamiltonian diffeomorphisms and Lagrangian submanifolds. Commun. Contemp. Math. 5:5 (2003), 803-811. Zbl 1053.53060 MR 2017719

[39] Y. Ostrover, Calabi quasi-morphisms for some non-monotone symplectic manifolds. Preprint, math.SG/0508090.

[40] A. Sard, Hausdorff measure of critical images on Banach manifolds. Amer. J. Math. 87 (1965), 158-174 and 760. Zbl 0137.42501 MR 0173748

[41] M. Schwarz, On the action spectrum for closed symplectically aspherical manifolds. Pacific J. Math. 193 (2000), 419-461. Zbl 1023.57020 MR 1755825

[42] C. Viterbo, Symplectic topology as the geometry of generating functions. Math. Ann. 292 (1992), 685-710. Zbl 0735.58019 MR 1157321

[43] J. von Neumann, Mathematical foundations of quantum mechanics, Princeton Landmarks in Mathematics, Princeton University Press, Princeton, NJ, 1955; translation of Mathematische Grundlagen der Quantenmechanik, Grundlehren Math. Wiss. 38, Springer-Verlag, Berlin 1932. Zbl 0064.21503 MR 1435976

[44] R. F. Wheeler, Quasi-measures and dimension theory. Topology Appl. 66 (1995), 75-92. Zbl 0842.28005 MR 1357876

Received October 31, 2004

Michael Entov, Department of Mathematics, Technion, Haifa 32000, Israel

E-mail: entov@math.technion.ac.il

Leonid Polterovich, School of Mathematical Sciences, Tel Aviv University, Tel Aviv 69978, Israel

E-mail: polterov@post.tau.ac.il 